\title{
An effective lower bound for a certain exponential function
}

Dedicated to Professor Yosizasu Eda on his 65-th birthday

1. In this note, we shall establish an effective lower bound for the function

$$
A_{1} e^{a_{1}}+\ldots+A_{K} e^{a_{k}} \text {, }
$$

where $A_{1}, \ldots, A_{k}$ are nonzero algebraic numbers, and $a_{1}, \ldots, a_{k}$ are distinct algebraic numbers. More precisely, we use the following notations. Let $\operatorname{deg} \alpha$ and $H(\alpha)$ denote, as usual, the degree and height of an algebraic number $\alpha$, respectively.

Using these notations, we put

$$
A=\max _{1 \leqslant i \leqslant k}\left(\operatorname{deg} A_{i}+H\left(A_{i}\right)\right) .
$$

Then we shall prove the following theorem.

THEOREMr. Let $d$ be an integer $\geqslant 2$. Then, for all distinct algebraic numbers $\alpha_{1}, \ldots, \alpha_{k}$ with degrees at most $d$, we have

$$
\left|A_{1} e^{a_{1}}+\ldots+A_{x_{i}} e^{a_{k_{k}}}\right|>\exp \left(-c_{1} H^{c_{2}}\right),
$$

where $H$ denotes the maximum of $H\left(a_{1}\right), \ldots, H\left(\alpha_{k}\right)$, and

$$
c_{1}=\exp \exp \left(7 k^{2} d^{k+1} A\right), \quad c_{2}=\exp \left(7 k^{2} d^{k+1} A\right) .
$$

Results of this kind were already obtained by Mahler [3] and Lang [2]. Mahler dealt only with the case when $a_{1}, \ldots, a_{k}$ are rational numbers. Lang treated the more general context of $E$-functions, but this is in a somewhat different direction to that studied by us.

I. should like to express my thanks to the referee for several helpful suggestions.

2. In this section, we shall prove three lemmas which will be needed in the sequel. We use the following notation. 
If $\xi$ is an algebraic number, we put

$$
\text { 闻 }=\max \left(\left|\xi^{(1)}\right|, \ldots,\left|\xi^{(h)}\right|\right),
$$

where $\xi^{(1)}, \ldots, \xi^{(h)}$ denote all the conjugates of $\xi^{(1)}=\xi$ over the rational number field.

Limanra 1. If $M_{i}(1 \leqslant i \leqslant p)$, as well as $N_{j}(1 \leqslant j \leqslant q)$ are nonzero algebraic numbers, and $\mu_{i}(1 \leqslant i \leqslant p)$, as well as $\lambda_{j}(1 \leqslant j \leqslant q)$ are distinct algebraic numbers, then the equality

$$
\left(\sum_{i=1}^{p} M_{i} e^{\mu_{i} x}\right)\left(\sum_{j=1}^{q} N_{j} e^{\lambda_{j} x}\right)=\sum_{h=1}^{l} R_{h} e^{\mathrm{e} h}, \quad \text { where } \varrho_{h}=\mu_{i}+\lambda_{j},
$$

where the terms having the same exponents have been combined and all $\varrho_{h}$ are distinct, has at least one nonzero $R_{h}$.

Proof. By Lindemann's theorem, we see that both of the sums in the left-hand side of (2) are different from zero. Thus their product is also nonzero, since the complex number field does not include any zerodivisors. This means that at least one nonzero $R_{h}$ appears in the right member of (2). This concludes the proof of the lemma.

LeMna 2. If $\alpha$ is an algebraic number, then

$$
\overline{|\alpha|}, \overline{\left|\alpha^{-1}\right|} \leqslant 2 H(\alpha) \text {. }
$$

Proof. We first prove the result for $\overline{|\alpha|}$. If $|\alpha| \leqslant 1$, the lemma is obvious, and hence we may assume $|\alpha|>1$. Denote by

$$
a_{0} x^{h}+a_{1} x^{h-1}+\ldots+a_{h}
$$

the minimal polynomial of $\alpha$, with the relatively prime rational integral coefficients. Then

$$
\begin{aligned}
\left|a_{0} \alpha^{h}\right| & =\left|a_{1} \alpha^{h-1}+\ldots+a_{h}\right| \leqslant H(\alpha)\left(|\alpha|^{h-1}+\ldots+1\right) \\
& =H(\alpha) \frac{|\alpha|^{h}-1}{|\alpha|-1}<H(\alpha) \frac{|\alpha|^{h}}{|\alpha|-1} .
\end{aligned}
$$

Hence

$$
|\alpha| \leqslant 1+H(\alpha) /\left|a_{0}\right| \leqslant 2 H(\alpha) .
$$

The proof when $\alpha^{-1}$ is completely similar. This concludes the proof of the lemma.

LFina 3. Let $\xi_{1}, \ldots, \xi_{l}$ be algebraic number's with the degrees and heights at most $D_{1}$ and $H_{1}$, respectively. Then,

$$
H\left(\xi_{1}+\ldots+\xi_{l}\right) \leqslant\left(32 l H_{1}^{l+1}\right)^{D_{1}^{l}}
$$

Proof. Denote by $a_{1}, \ldots, a_{l}$, the leading coefficients of the minimal polynomials of $\xi_{1}, \ldots, \xi_{l}$, respectively, with rational integral coefficients. Then

$$
\left(a_{1} \ldots a_{l}\right)^{D_{1}^{l}} \prod_{i_{1}, \ldots, i_{l}}\left[x-\left(\xi_{1}^{\left(i_{1}\right)}+\xi_{2}^{\left(i_{2}\right)}+\ldots+\xi_{l}^{\left(i_{l}\right)}\right)\right]
$$

where the product is taken over all the respective conjugates of $\xi_{1}^{(1)}=\xi_{1}, \ldots, \xi_{l}^{(1)}=\xi_{l}$, is a polynomial witll rational integral coefficients. Furthermore, it is clear that the minimal polynomial of $\xi_{1}+\ldots+\xi_{l}$ is a factor of this polynomial, and hence by Gelfond's book ([1], p. 14, Lemma IV) and Lemma 2, we have

$$
\begin{aligned}
H\left(\xi_{1}+\ldots+\xi_{l}\right) & \leqslant\left(a_{1} \ldots a_{l}\right)^{D_{1_{2}}^{l} D_{1}^{l}} \max \left(1, \overline{\left.\xi_{1}+\ldots+\left.\xi_{l}\right|^{D_{1}^{l}}\right) 2^{3 D_{1}^{l}}}\right. \\
& \leqslant H_{1}^{l D_{1_{2}}^{l} D_{1}^{l}}\left(2 l H_{1}\right)^{D_{1_{2}}^{l} D_{1}^{l}}=\left(32 l H_{1}^{l+1}\right)^{D_{1}^{l}}
\end{aligned}
$$

This concludes the proof of the lemma.

3. In this section, we shall establish the modified form of the exponential fanction in the theorem by using the argument in Gelfond's book ([1], pp. $4 \tilde{b}-47$ ). We first note that if $k=1$, then the theorem is obvious. Hence we may assume

$$
k \geqslant 2
$$

By (1) and Lemma 2, we have

$$
\overline{\mid A_{i}} \mid \leqslant 2 A \quad(1 \leqslant i \leqslant 7)
$$

Let $a$ be the smallest positive rational integer such that all the $a A_{i}$ axe algebraic integers. Hence

$$
a \leqslant A^{k}
$$

Put $K_{0}=\boldsymbol{Q}\left(A_{1}, \ldots, A_{k}\right)$, where $\boldsymbol{Q}$ is, as usual, the rational number field. Let $\nu_{0}$ be the degree of the extension $K_{0} / Q$. Hence

$$
\nu_{0} \leqslant A^{k}
$$

Denote by $A_{i}^{(q)}$ an element which is conjugate to $A_{i}^{(1)}=A_{i}$ over the rational number field. Then the coefficients $B_{h_{1} \ldots J_{i k}}$ in the product

$$
a^{y_{0}} \prod_{q=1}^{v_{0}}\left(\sum_{i=1}^{k} A_{i}^{(q)} x_{i}\right)=\sum_{h_{1}+\ldots+h_{k}=\nu_{0}} B_{h_{1} \ldots h_{k}} x_{1}^{h_{1}} \ldots x_{k}^{h_{k}}
$$

will be rational integers, since all the $a A_{i}^{(q)}$ are algebraic integers and all the $B_{h_{1} \ldots h_{k} \text {, }}$ are symmetric functions of the roots of the irreducible equation which is satisfied by a primitive element of the extension $K_{0} / Q$. 
Setting $x_{i}=e^{a_{i}}\left(1 \leqslant i \leqslant 7_{i}\right)$ into identity $(6)$, we obtain

$$
a^{\mathbf{y}_{0}} \prod_{q=1}^{y_{0}}\left(\sum_{i=1}^{k} A_{i}^{(q)} e^{\alpha_{i}}\right)=\sum_{j=1}^{m} B_{j} e^{\beta_{j}}
$$

where all the $\beta_{j}$ are algebraic and distinct, and all the $B_{j}$ are nonzero rational integers. It follows by Lemma 1 that $m \geqslant 1$. Further by (3), (4) and Lemma 2, we have

$$
\left|a \sum_{i=1}^{k} A_{i}^{(q)} e^{a} i\right| \leqslant 2 k A^{k+1} e^{2 Z}
$$

Next, wo shall estimate $m, B_{j}, \operatorname{deg} \beta_{j}, H\left(\beta_{j}\right)$. It follows easily by $(5)$ and (6) that

$$
m \leqslant\left(\begin{array}{c}
\nu_{0}+k-1 \\
k-1
\end{array}\right) \leqslant\left(\nu_{0}+1\right)^{k-1} \leqslant 2^{k} A^{k^{2}}
$$

We also obtain

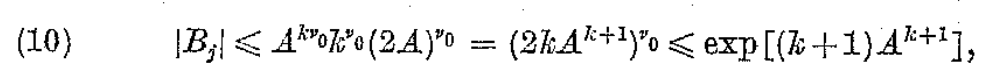

by (i). Moreover, since

$$
\text { (11) } \quad \beta_{j}=h_{1} \alpha_{1}+\ldots+h_{k} \alpha_{k}, \quad h_{1}+\ldots+h_{k i}=v_{0},
$$

we have

$$
\operatorname{deg} \beta_{j}^{\top} \leqslant d^{h} .
$$

It remains to estimate $H\left(\beta_{j}\right)$. We first note by (11) that

Hence by Lemma 3, we have

$$
\begin{gathered}
H\left(h_{i} \alpha_{i}\right) \leqslant v_{0}^{d} H \leqslant A^{k d} H \quad(i=1, \ldots, k), \\
\operatorname{deg}\left(h_{i} \alpha_{i}\right) \leqslant d \quad(i=1, \ldots, k) .
\end{gathered}
$$

$$
\begin{aligned}
H\left(\beta_{j}\right) & =H\left(h_{1} \alpha_{1}+\ldots+h_{k} \alpha_{k}\right) \leqslant\left[32 \hbar\left(A^{k d} H\right)^{k+1}\right]^{d^{k}} \\
& \leqslant \exp \left[k\left(k_{i}+1\right) d^{k+1} A\right] H^{(k+1) d^{k}} .
\end{aligned}
$$

Sow, we go on with our arguments. Put $K_{1}=\boldsymbol{Q}\left(\beta_{1}, \ldots, \beta_{m}\right)$. Then by (11), we have $K_{1} \subset \boldsymbol{Q}\left(a_{1}, \ldots, \alpha_{k}\right)$. Let $\nu_{1}$ be the degree of the extension $K_{1} / Q$. Hence

$$
\nu_{1} \leqslant d^{k}
$$

Denoting by $\beta_{j}^{(q)}\left(q=1, \ldots, v_{1}\right)$ all the images of $\beta_{j}^{(1)}=\beta_{j}$ under $\nu_{1}$ isomorphisms of $K_{1}$ over $Q$, we shall consider the product

$$
\prod_{q=1}^{\eta_{1}}\left(\sum_{j=1}^{m} B_{j} e^{\beta_{j}^{(q)}}\right)=\sum_{h=1}^{s} G_{h} e^{\gamma_{h}}
$$

where all the $\gamma_{h}$ are algebraic and distinct, and all the $C_{h}$ are nonzero rational integers. As before, we see that $s \geqslant 1$. Further we note that if an algebraic power $\gamma_{h}$ appears in (15), then all the conjugates of $\gamma_{h}$ over $Q$ also appear as powers in the right member of (15) with the same $C_{h} \neq 0$. Indeed, this is a direct consequence of the fact that $\gamma_{h}$ is linear form of all the roots of the irreducible equation which is satisfied by a primitive element of the extension $K_{1} / Q$ and all the conjugates of $\gamma_{b}$ are obtained by suitable permutations of these roots. We also hare

$$
\left|\beta_{j}^{(q)}\right| \leqslant 2 \nu_{0} H \leqslant 2 A^{k} H
$$

by (11). Hence it follows by (9) and (10) that

$$
\left|\sum_{j=1}^{m} B_{j} e^{p_{j}^{(q)}}\right| \leqslant m \exp \left[(k+1) A^{k+1}\right] e^{2 A^{k_{H}}} \leqslant \exp \left[(k+1)^{2} A^{k+1}\right] e^{2 A^{k_{H}}} .
$$

Finally we shall estimate of $s, C_{h}$, deg $\gamma_{h}$ and $H\left(\gamma_{h}\right)$. From (14) and (15), we have easily

$$
s \leqslant m^{a^{k}} \leqslant\left(2 A^{k}\right)^{k d^{k}} \leqslant \exp \left(k^{2} d^{k} A\right) .
$$

We also obtain

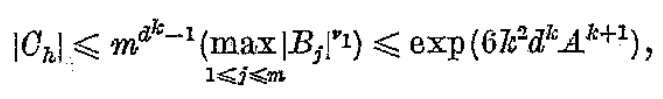

by (15). Next, since

we have

$$
\gamma_{h}=\beta_{h_{I}}^{(1)}+\beta_{h_{2}}^{(2)}+\ldots+\beta_{h_{1}}^{\left(\nu_{1}\right)}, \quad 1 \leqslant h_{l} \leqslant m,
$$

$$
\operatorname{deg} \gamma_{h} \leqslant(\alpha ! !)^{k},
$$

by (11). For, $\gamma_{h}$ is included in the smallest Galois extension of $Q\left(a_{1}, \ldots, \alpha_{k}\right)$. Furthermore, by (12), (13), (18) and Lemma 3, we have

$$
\begin{aligned}
H\left(\gamma_{h}\right) & \leqslant\left\{32 \nu_{1}\left(\exp \left[k(k+1) d^{k+1} A\right] H^{(k+1) d^{k}}\right) p_{1}+1\right\}^{d^{k \nu_{1}}} \\
& \leqslant \exp \left(\widetilde{a} l^{2} d^{2 k d^{k}} A\right) H^{4 k d^{2 k d^{k}}},
\end{aligned}
$$

since $k \geqslant 2, a \geqslant 2$ and $\nu_{1} \leqslant d^{k}$.

4. From the arguments of Section 3 , we may assume our exponential function in the theorem to be of the form

$$
\sigma_{1} \sum_{i=1}^{d_{1}} e^{\gamma_{1}^{(i)}}+\sigma_{2} \sum_{i=1}^{a_{2}} e^{\gamma_{2}^{(i)}}+\ldots+\sigma_{n} \sum_{i=1}^{a_{n}} e^{\gamma_{n}^{(i)}}
$$

where $C_{1}, \ldots, O_{n}$ are nonzero rational integers and $\gamma_{1}^{(\mathrm{i})}=\gamma_{1}, \ldots, \gamma_{n}^{(1)}=\gamma_{n}$ are pairwise-nonconjugate algebraic numbers and $\gamma_{l}^{(i)}\left(i=1, \ldots, d_{l}\right)$ are all the conjugates of $\gamma_{l}$ over the rational number field, and $d_{1}, \ldots, d_{n}$ are the degrees of $\gamma_{1}, \ldots, \gamma_{n}$, respectively. Moreover, from the last para- 
graph of Section 3, we see that

$$
\begin{gathered}
s=d_{1}+\ldots+d_{n}, \\
\max _{1 \leqslant l \leqslant n}\left|O_{l}\right| \leqslant \exp \left(6 k^{2} d^{z} A^{k+1}\right) .
\end{gathered}
$$

Put

$$
\hat{D}=(d !)^{k}, \quad \hat{H}=\exp \left(5 k^{2} d^{2 k d^{k}} A\right) H^{4 k d^{2 k} d^{k}} .
$$

Then, from the last paragraph of preceding section, we also see that

$$
\max \left(d_{1}, \ldots, d_{n}\right) \leqslant \hat{D}, \quad \max \left(H\left(\gamma_{1}\right), \ldots, H\left(\gamma_{n}\right)\right) \leqslant \hat{H}
$$

Now, we note first that if $s=1$, then the theorem is obvious. Hence we may assume $s \geqslant 2$. It follows immediately by $(20)$ that

$$
\hat{D} \geqslant 4, \quad \hat{H} \geqslant 16,
$$

since $k \geqslant 2$. We take a number $r$ to be any large positive integer which will be explicitly determined below. Put

$$
N+1=s(r+1)
$$

Let $D$ be the differential operator

$$
D=\frac{d}{d z}
$$

Now, according to Siegel's book ([5], pp. 12-15), we can determine $s$ polynomials $P_{l, i}(z)\left(l=1, \ldots, n ; i=1, \ldots, d_{l}\right)$ of degrees $r$ such that the function

$$
F(z)=\sum_{l=1}^{n} \sum_{i=1}^{a_{l}} P_{l, i}(z) e^{\gamma_{l}^{(i)_{z}}}
$$

vanishes at $z=0$ of order $N$. We obtain an explicit formula for $P_{l, i}(z)$ :

$$
\begin{array}{r}
P_{l, i}(z)=\prod_{\substack{p=1 \\
p \neq i}}^{d_{l}}\left(D+\gamma_{l}^{(i)}-\gamma_{l}^{(p)}\right)^{-r-1} \prod_{\substack{h=1 \\
h \neq l}}^{n} \prod_{\substack{q=1 \\
p_{h}}}^{d_{h}}\left(D+\gamma_{l}^{(i)}-\gamma_{h}^{(q)}\right)^{-r-1} \frac{z^{r}}{r !} \\
\left(l=1, \ldots, n ; i=1, \ldots, d_{l}\right)
\end{array}
$$

(see $[5]$, p. 15). From (24), we see that the power series of $F^{\prime}(z)$ takes the form

$$
F(z)=\frac{z^{N}}{N !}+\ldots
$$

Moreover, it can be shown that $F(z)$ has an integral representation of the form

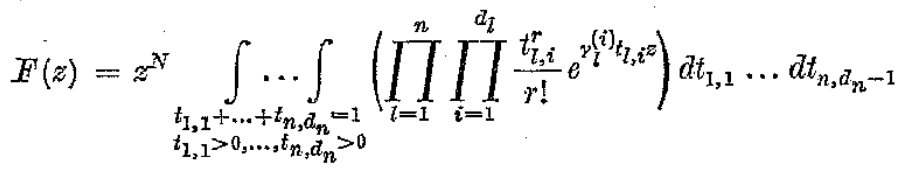

(see [5], p. 26). By comparing (26) and (27), it follows immediately that the integral in (27) has for $z=0$ the value $1 / N$ !; henee for any complex number $z$, we have

$$
|F(z)| \leqslant \frac{|z|^{N} e^{\lambda|\varepsilon|}}{N !}, \quad \text { where } \lambda=\max \left(\left|\overline{\gamma_{1}}\right|, \ldots,\left|\overline{\gamma_{n 1}}\right|\right)
$$

From the above formula $(25)$, it is clear that all the $P_{l, i}(z)(l=1, \ldots, n$; $i=1, \ldots, d_{l}$ ) are nonzero polynomials of degrees $r$.

5. In this section, we shall use the argument in Mrahler's paper [4]. Put

(29) $\quad P_{l_{i}, i, j}(z)=\left(D+\gamma_{l}^{(i)}\right)^{j} P_{l, i}(z) \quad\left(l=1, \ldots, n ; i=1, \ldots, d_{l}\right.$;

$$
j=0,1,2, \ldots) \text {, }
$$

where $P_{l, i}(z)$ have been constructed in the preceding section. We begin by proving the following lemma.

LEMMA 4 (Mahler). The determinant

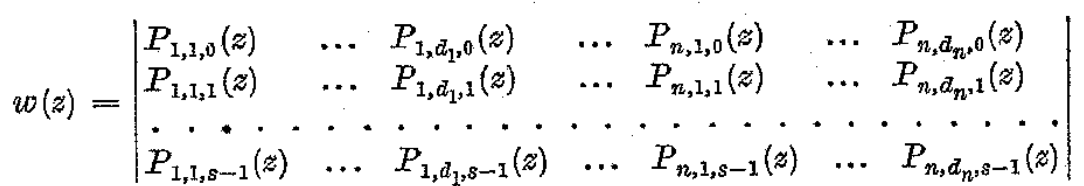

is not identically zero. Moreover, w(z) can be written

$$
w(z)=z^{v} R(z)
$$

where $v=N-s(s-1) / 2$, and $R(z)$ is a nonzero polynomial with degree at most $(s-1)(s-2) / 2$.

Proof. We first note that the $s$ exponential functions

$$
Q_{l, i}(z)=P_{l, i}(z) e^{\gamma_{l}^{(i)}} \quad\left(l=1, \ldots, n ; i=1, \ldots, d_{l}\right)
$$

are Jinearly independent over the complex number field, so that the Wronskian

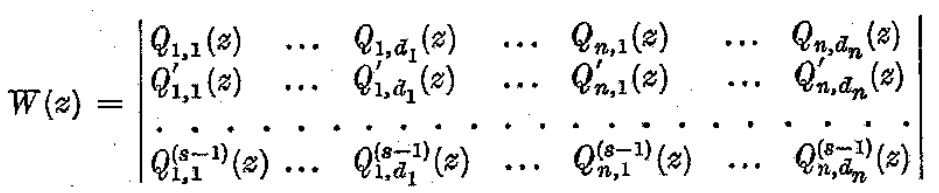


does not vanish identically. By a well known symbolic relation, we have

$$
\begin{aligned}
Q_{l, i}^{(j)}(z)=\left(\frac{d}{d z}\right)^{j}\left(P_{l, i}(z) e^{\gamma_{l}^{(i)} z}\right)=e^{\gamma_{l}^{(i)} z}\left(D+\gamma_{l}^{(i)}\right)^{j} P_{l, i}(z) \\
\\
\quad\left(l=1, \ldots, n ; i=1, \ldots, d_{l} ; j=0,1,2, \ldots\right) .
\end{aligned}
$$

Therefore, it follows by (29) that

$$
Q_{l, i}^{(j)}(z)=P_{l, i, j}(z) e^{\gamma_{l}^{(i)_{z}}} \quad\left(l=1, \ldots, \eta ; i=1, \ldots, d_{l} ; j=0,1,2, \ldots\right) .
$$

On substituting these into (30), we obtain

$$
W(z)=\left(\prod_{l=1}^{n} \prod_{i=1}^{a_{l}} e^{\gamma_{l}^{(i)}}\right)^{z} w(z)
$$

and hence it follows that $w(z)$ is not identically zero. Next, in the determinant $w(z)$ multiply, for $i=1, \ldots, d_{1}$, the $i$ th column by the factor $e^{\gamma_{1}^{(i)}}$, and for $i=1, \ldots, d_{2}$, the $\left(d_{1}+i\right)$ th column by the factor $e^{\gamma_{2}^{(i)_{3}}}$ and so on. Finally add the 2 th, 3 th, ..., sth new columns to the first new column. This leads to the equation

$20(z) e^{\gamma_{1} z}$

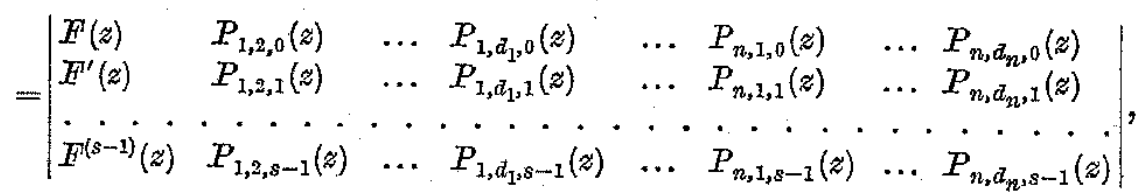

since

$$
F^{(j)}(z)=\sum_{l=1}^{n} \sum_{i=1}^{d_{l}} P_{l, i, j}(z) e^{\gamma_{l}^{(i)_{z}}} \quad(j=0,1,2, \ldots) .
$$

On multiplying in this determinant the successive rows by the factors $1, z, \ldots, z^{s-1}$ respectively, we arrive at the equation

$$
z^{s(s-1) / 2} w(z) e^{y_{1}}
$$

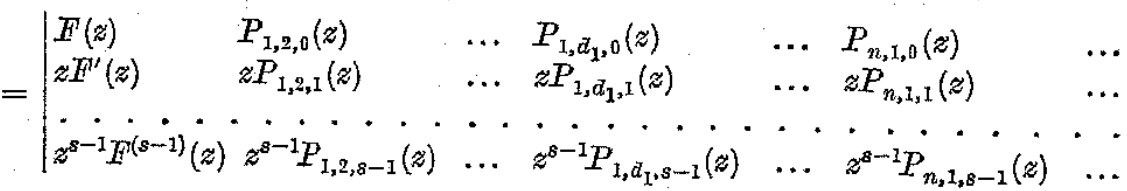

$$
\begin{aligned}
& \text { ‥ } P_{n, a_{n}, 0}(z) \\
& \ldots z P_{n, d_{n}, 1}(z) \\
& \text { - } \cdot \cdot \cdot \cdot \cdot \cdot \cdot
\end{aligned}
$$

Hence by $(26), w(z)$ itself vanishes at $z=0$ of order at least

Hence the polynomial

$$
N-s(s-1) / 2=v \text {. }
$$

$$
R(z)=w(z) / z^{v}
$$

has degree at most $s r-v=(s-1)(s-2) / 2$, since $w(z) \neq 0$ has degree at most $s r$. This concludes the proof of the lemma.

Now the polynomials $P_{l, i, j}(z)$ have been defined by the equation (29). These equations show that they have algebraic coefficients, hence that the values $P_{l, i, j}(1)$ are algebraic numbers. In terms of these polynomials, the derivatives

$$
F^{(j)}(z)=\sum_{l=1}^{n} \sum_{i=1}^{d_{l}} P_{l, i, j}(z) e^{\gamma_{l}^{(i)_{z}}} \quad(j=0,1,2, \ldots)
$$

are linear forms in the $s$ exponential functions $e^{\gamma_{l}^{(i)} z}(\eta=1, \ldots, n$; $\left.i=1, \ldots, d_{l}\right)$. By Lemma 4 , the determinant $w(z)$ of the first $s$ of these linear forms is not identically zero and vanishes at $z=1$ of order at most $(s-1)(s-2) / 2=t$ (say). Suppose that it in fact vanishes at $z=1$ of exact order $\tau$, so that

$$
w(1)=w^{\prime}(1)=\ldots=v^{(z-1)}(1)=0, \quad w^{(\tau)}(1) \neq 0
$$

On solving the first $s$ linear forms

$$
\text { where } 0 \leqslant \tau \leqslant t \text {. }
$$

$$
F^{(j)}(z)=\sum_{l=1}^{n} \sum_{i=1}^{d_{l}} P_{l, i, j}(z) e^{\gamma_{l}^{(i)} z} \quad(j=0,1, \ldots, s-1)
$$

for $e^{\gamma_{l}^{(i)}}\left(l=1, \ldots, n ; i=1, \ldots, d_{l}\right)$ respectively, we have

$$
w(z) e^{\gamma_{l}^{(i)_{z}}}=\sum_{j=0}^{s-1} u u_{l, i, j}(z) F^{(i)}(z) \quad\left(l=1, \ldots, n ; i=1, \ldots, a_{l}\right),
$$

where the $u_{7, i, j}(z)$ are cofactors of the determinant $w(z)$ and hence are again polynomials with algebraic coefficients. On differentiating these $s$ : equations $\tau$ times, we obtain

$$
\sum_{h=0}^{\tau}\left(\begin{array}{l}
\tau \\
h
\end{array}\right) w^{(h)}(z)\left(\gamma_{l}^{(i)}\right)^{\tau-h} e^{\gamma_{l}^{(i)} z}=\sum_{j=0}^{s+\tau-1} U_{l, i_{j},}(z) F^{(j)}(z)
$$

where the $U_{l, i, j}(z)$ also are polynomials with algebraic coefficients. Here finally put $z=1$. Then it follows by (31) that

$$
w^{(t)}(1) e^{y_{l}^{(i)}}=\sum_{j=0}^{s+t-1} U_{l, i, j}(1) F^{(i)}(1) \quad\left(l=1, \ldots, n ; i=1, \ldots, d_{l}\right) .
$$


The $s+\tau$ expressions

$$
F^{(j)}(1)=\sum_{l=1}^{n} \sum_{i=1}^{a_{l}} P_{l, i, j}(1) e^{\gamma_{l}^{(i)}} \quad(j=0,1, \ldots, s+\tau-1)
$$

on the right-hand sides of the equations are linear forms in $e^{y_{l}^{(i)}}(l=1, \ldots$ $\left.\ldots, n ; i=1, \ldots, d_{l}\right)$ with algebraic coefficients. Since $v^{(i)}(1) \neq 0$, these $s+\tau$ linear forms can be solved for each of the $e^{\gamma_{l}^{(i)}}$. It follows that there exist $s$ distinct suffices $J=J(1), J(2), \ldots, J(s)$ in the interval $0 \leqslant J$ $\leqslant s+t-1=s(s-1) / 2$ for which the corresponding linear forms

$$
F^{(J(j))}(1)=\sum_{i=1}^{n} \sum_{i=1}^{a_{l}} P_{l, i, J(j)}(1) e^{\gamma_{l}^{(i)}} \quad(j=1, \ldots, s)
$$

in $e^{y_{l}^{(i)}}\left(l=1, \ldots, n ; i=1, \ldots, d_{l}\right)$ are linearly independent. Hence the determinant of these forms

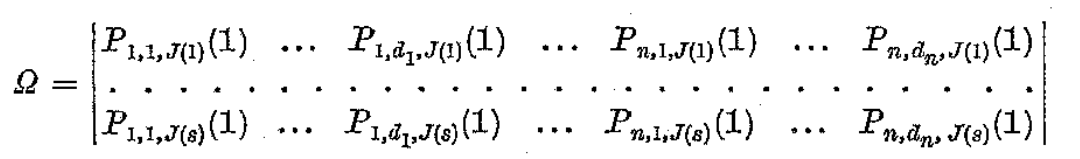

is distinct from zero.

6. In this section, we shall first find an upper estimate for $\left|P_{l, i, J(j)}(1)\right|$. We use the following notation. Let

$$
F_{1}(z)=\sum_{i} a_{i} z^{i}
$$

be a polynomial with complex coefficients and let

$$
F_{2}(z)=\sum_{i} b_{i} z^{i}
$$

be a polynomial with real coefficients $\geqslant 0$. We write $F_{1}(z) \prec F_{2}(z)$ if $\left|a_{i}\right| \leqslant b_{i}$ for all $i$. Using this notation and applying an operator identity

$$
(\omega+D)^{-r-1}=\omega^{-r-1} \sum_{\varrho \Rightarrow 0}^{\infty}\left(\begin{array}{c}
-r-1 \\
\varrho
\end{array}\right) \omega^{-\varrho} D^{\varrho} \quad(\omega \neq 0)
$$

we obtain easily

(33) $(\omega+D)^{-r-1} z^{h} \prec|\omega|^{-r-1} \sum_{\varrho=0}^{\infty}\left(\begin{array}{c}-r-1 \\ \varrho\end{array}\right)|\omega|^{-\varrho}(-D)^{\varrho} z^{h}=(|\omega|-D)^{-r-1} z^{h}$
Let $I I$ denote the maximum of the $s(s-1) / 2$ numbers

$$
\begin{gathered}
\frac{1}{\left|\gamma_{l}^{(i)}-\gamma_{l}^{(j)}\right|} \quad\left(t=1, \ldots, n ; 1 \leqslant i<j \leqslant d_{l}\right), \\
\frac{1}{\left|\gamma_{l}^{(i)}-\gamma_{h}^{(j)}\right|} \quad\left(1 \leqslant l<h \leqslant n ; \quad i=1, \ldots, d_{l} ; j=1, \ldots, d_{h}\right) .
\end{gathered}
$$

Then it follows by (23), (25) and (33) that

$$
P_{l, i}(z) \prec\left(M I^{-1}-D\right)^{r-N} \frac{z^{r}}{r !} \quad\left(l=1, \ldots, n ; i=1, \ldots, d_{l}\right) .
$$

Moreover, we have

$$
\left(M I^{-1}-D\right)^{r-N} \frac{z^{r}}{r !}=M^{N-r} \sum_{\varrho=0}^{r}\left(\begin{array}{c}
N-r+\varrho-1 \\
\varrho
\end{array}\right) M^{e} D^{\varrho} \frac{z^{r}}{r !} \prec \sum_{\varrho=0}^{r}\left(\begin{array}{c}
N \\
\varrho
\end{array}\right) M^{N-r+e} z^{r-\varrho} .
$$

Hence the heights of $P_{l, i}(z)$ (that is, the maximum of the absolute values of its coefficients) are at most

$$
2^{N} \mathbb{H}^{N}=(2 M)^{N} \text {. }
$$

On the other hand, it follows by (21), Lemma 2 and Lemma 3 that

Put now

$$
M \leqslant 2(2 \hat{H})^{3 \hat{D}^{2}}=2^{1+3 \hat{D}^{2}} \hat{H}^{3 \hat{D}^{2}}
$$

Then it follows by (22) and (29) that the heights of $P_{l, i, J(j)}(z)$ are at most

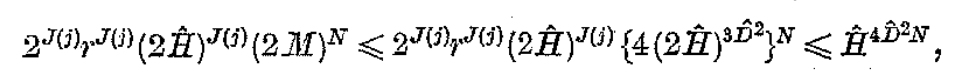

since $J(j) \leqslant s(s-1) / 2$. Hence we obtain

$$
\begin{aligned}
& \text { (35): }\left|P_{l, i, J(j)}(1)\right| \leqslant \hat{H}^{4 \hat{D}^{2} N}(\underbrace{1+1+\ldots+1}_{r}) \leqslant \hat{H}^{5^{2} N} \\
& \left(l=1, \ldots, n ; i=1, \ldots, d_{l} ; j=1, \ldots, s\right),
\end{aligned}
$$

by (23). Next, we choose a positive integer $T$ for which the $s(s+1) / 2$ numbers

$$
\begin{gathered}
T_{l}^{(i)} \quad\left(l=1, \ldots, n ; i=1, \ldots, d_{l}\right), \\
\frac{T}{\left(\gamma_{l}^{(i)}-\gamma_{l}^{(j)}\right)} \quad\left(l=1, \ldots, n ; 1 \leqslant i<j \leqslant d_{l}\right), \\
\frac{T}{\left(\gamma_{l}^{(i)}-\gamma_{h}^{(j)}\right)} \quad\left(1 \leqslant l<h \leqslant n ; i=1, \ldots, d_{l} ; j=1, \ldots, d_{h}\right)
\end{gathered}
$$


all are algebraic integers. By (21) and Lemma 3, we can take

$$
T \leqslant \hat{H}^{n}\left\{(4 \hat{H})^{3 \hat{D}^{2}}\right\}^{s(s-1) / 2} \leqslant(4 \hat{H})^{2 s^{2} \hat{D}^{2}}
$$

since $n \leqslant s$. It follows from $(23)$ and $(25)$ that the polynomials

$$
r ! T^{N} P_{l, i}(z)
$$

have algebraic integral coefficients. Hence the polynomials

$$
r ! T^{N+J(j)} P_{l, i, J(j)}(z)=\left(T D+T \gamma_{l}^{(i)}\right)^{J(j)} r ! T^{N} P_{l, i}(z)
$$

also have algebraic integral coefficients. Put now

$$
\begin{aligned}
& q_{l, i, j}=r ! T^{N+J(j)} P_{l, i, J(j)}(1) \quad\left(l=1, \ldots, n ; i=1, \ldots, d_{l} ;\right. \\
& j=1, \ldots, s) \text {. }
\end{aligned}
$$

Then all the numbers $q_{7, i, j}$ are algebraic integers, and from the last paragraph of Section 5 , their determinant is different from zero, namely

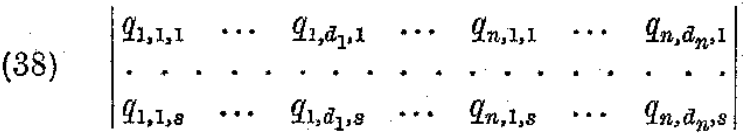

$$
\begin{aligned}
& =(r !)^{s} T^{s N+J(\mathrm{I})+\ldots+J(s)} \Omega \neq 0 .
\end{aligned}
$$

Furthermore by (35), (36) and (37), we have

(39) $\quad\left|q_{l, i, j}\right| \leqslant r ! T^{N+J(j)}\left|P_{l, i, J(j)}(1)\right| \leqslant r !\left\{(4 \hat{H})^{\left.2 s^{2} \hat{D}^{2}\right\}}\right\}^{N+J(j)} \hat{H}^{5 \hat{D}^{2} N} \leqslant r ! \hat{H}^{8 s^{2} \hat{D}^{2} N}$

$$
\left(l=1, \ldots, n ; i=1, \ldots, d_{l} ; j=1, \ldots, s\right),
$$

since $J(j) \leqslant s(s-1) / 2$. Now, in analogy to the algebraic integers $q_{l, i, j}$ put

$$
L_{j}=r ! T^{N+J(j)} F^{(J(j))}(1) \quad(j=1, \ldots, s) .
$$

Hence it follows by (32) and (37) that $L_{j}$ is the linear form

$$
L_{j}=\sum_{l=1}^{n} \sum_{i=1}^{d_{l}} q_{l, i, j} e^{\gamma_{l}^{(i)}} \cdots(j=1, \ldots, s)
$$

in $e^{\gamma_{l}^{(i)}}\left(l=1, \ldots, n ; i=1, \ldots, d_{l}\right)$. From $(38), x_{1}, \ldots, L_{s}$ are linearly independent. We shall find un upper bound for $\left|L_{j}\right|$. We first estimate $\left|F^{(J(j))}(1)\right|$. By Oauchy's integral formula, we have

$$
F^{(J(j))}(1)=\frac{J(j) !}{2 \pi i} \int_{\Gamma} \frac{F(z)}{(z-1)^{J(j) \div 1}} d z,
$$

where $\Gamma$ is the circle $|z|=2$, A well known form of Stirling's formula states that

$$
N !=\sqrt{2 \pi N} N^{N} e^{-N+\eta(N)}, \quad \text { where } 0<\eta(N)<1 / 12 N .
$$

Hence it follows immediately by (21) and (28) that

$$
\left|F^{(J(j))}(1)\right| \leqslant e^{2 N+4 \hat{H}-N \log N} .
$$

Thus we obtain

$$
\left|L_{j}\right| \leqslant r ! e^{3 s^{2} \hat{D}^{2} N \log \hat{H}+4 \hat{H}-N \log N}
$$

7. In this section, we shall conclude the proof of the theorem. Put $K=Q\left(\gamma_{1}^{(1)}, \ldots, \gamma_{1}^{\left(d_{1}\right)}, \ldots, \gamma_{n}^{(1)}, \ldots, \gamma_{n}^{\left(d_{n}\right)}\right)$. Let $\nu$ denote the degree of the extension $K / Q$. Let $\sigma$ be an isomorphism of $K / Q$ which leaves every element of $\boldsymbol{Q}$ fixed. Let $\theta$ be an element of $K$. We shall often write $\theta^{\sigma}$ instead of $\sigma(\theta)$ which is the image of $\theta$ under the isomorphism $\sigma$. Furthermore, we use the following notation.

From the first paragraph of Section 4 , we agree that an element $\gamma_{l}^{(i)^{\sigma}}$ is equal to exactly one among $\gamma_{l}^{(1)}, \ldots, \gamma_{l}^{\left(d_{l}\right)}$. Hence we put

$$
\gamma_{l}^{(i)^{\sigma}}=\gamma_{l}^{\left([\sigma]_{l}\right)} \quad\left(l=1, \ldots, n ; i=1, \ldots, d_{l}\right) .
$$

Thus $[\sigma i]_{l}$ is equal to exactly one among the numbers $1,2, \ldots, d_{l}$. Using these notations and recalling the explicit formulas $(25)$ for $P_{l, i}(z)$, we see easily that the equalities

$$
P_{l, i}^{\sigma}(1)=P_{l,[a i]_{l}}(1) \quad\left(l=1, \ldots, n ; i=1, \ldots, d_{l}\right)
$$

hold. Moreover from (29), we have

$$
P_{l, i, J(j)}^{\sigma}(1)=P_{l,[\sigma i]_{l}, J(j)}(1) \quad\left(l=1, \ldots, n ; i=1, \ldots, d_{l} ; j=1, \ldots, s\right) .
$$

Therefore we also see by (37) that the equalities

$$
\text { (43) } \quad q_{l, i, j}^{\sigma}=q_{l,[\sigma i]_{l}, j} \quad\left(l=1, \ldots, n ; i=1, \ldots, d_{l} ; j=1, \ldots, s\right)
$$

hold. Next, put

$$
I=C_{1} \sum_{i=1}^{d_{1}} e^{\gamma_{1}^{(i)}}+\ldots+C_{n} \sum_{i=1}^{d_{n}} e^{\gamma_{n}^{(i)}}
$$

which is not equal to zero. Hence $L$ is linearly independent of certain $s-1$ of the forms $L_{j}$. We may suppose, without loss of generality that the $s$ forms

$$
L, L_{2}, \ldots, L_{s}
$$


are linearly independent. Hence their determinant

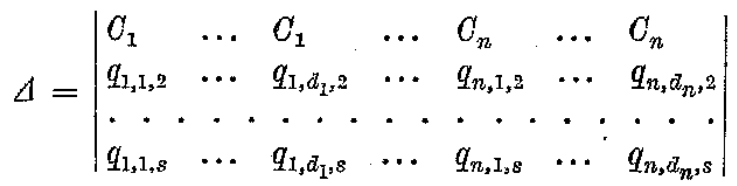

is distinct from zero. This determinant is an algebraic integer, and so (46)

$$
\left|\operatorname{Norm}_{\mathbb{K} / Q}(\Delta)\right| \geqslant 1 \text {. }
$$

Now noting $\sigma_{l}^{\sigma}=C_{l}(l=1, \ldots, n)$, we have

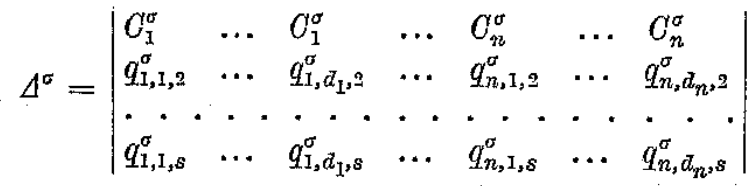

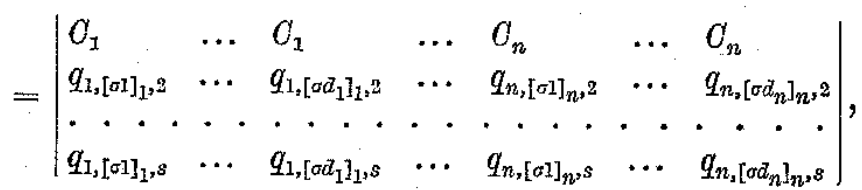

by (43). Here we note that, by change of columns, we have

$$
\Delta^{\sigma}= \pm \Delta
$$

since $[\sigma 1]_{1}, \ldots,\left[\sigma d_{1}\right]_{1}$ is just a permutation of $1, \ldots, d_{1}$. Thus

$$
\left|\operatorname{Norm}_{K / Q}(\Delta)\right|=|\Delta|^{2} \text {. }
$$

Hence by (46), we obtain

$$
|\Delta| \geqslant 1 \text {. }
$$

Now, in the right-hand side of $(45)$, multiply for $i=1, \ldots, d_{1}$, the ith column by the factor $e^{\gamma_{1}^{(i)}}$, and for $i=1, \ldots, d_{2}$, the $\left(d_{1}+i\right)$ th column by the factor $e^{\gamma_{2}^{(i)}}$,

the first $e$, and so on. Finally, add 2 th, 3th, ..., sth new columns

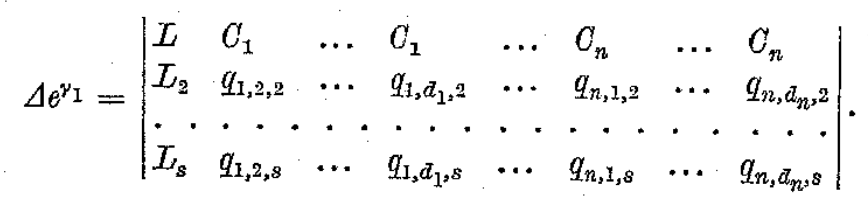

Hence by (34), (39) and (42), we have

(48) $\left|\Delta e^{\gamma_{1}}\right| \leqslant s !\left\{\left[L \mid\left(r ! \hat{H}^{8 s^{2} \hat{D}^{2} N}\right)^{s-1}+\left(\max _{j}\left|L_{j}\right|\right)\left(\max _{l}\left|O_{l}\right|\right)\left(\gamma ! \hat{H}^{8 s^{2} \hat{D}^{2} N}\right)^{s-2}\right\}\right.$

$$
\leqslant|L|(r !)^{s} \hat{H}^{s s^{2}(s-1) \hat{D}^{2} N}+(r !)^{s-1} \hat{H}^{8 s^{2}(s-1) \hat{D}^{2} N}\left(\max \left|C_{l}\right|\right) e^{4 \hat{H}-N \mid \log N} .
$$

From (23), $N \geqslant r s$. Hence we obtain

$$
(r !)^{s-1} e^{-N \log N} \leqslant e^{(s-1) r \log r-r s \log r}=e^{-r \log r} .
$$

Moreover, by (19), (20) and (34), we have

$$
\begin{aligned}
& 8 s^{2}(s-1) \hat{D}^{2} N \log \hat{H}+\log \left(\max _{l} ; \sigma_{l} \mid\right)+4 \hat{H} \\
& \leqslant 8 s^{2}\left(s^{2}-1\right) \hat{D^{2}} r \log \hat{H}+6 k^{2} d^{k} A^{k+1}+4 \hat{H} \\
& \leqslant 8 s^{4} \hat{D}^{2} r \log \hat{H}=\frac{8}{9} r \log r
\end{aligned}
$$

since $N \leqslant(s+1) r$. Eence it follows from (47) and (48) that

$$
\left.\left|e^{\gamma_{1}}\right| \leqslant \mid L\right](r !)^{s} \hat{B}^{8 s^{2}(s-1) \hat{D}^{2} N}+e^{-\frac{1}{9} r \log r} .
$$

On the other hand, it is clear that

$$
\left|e^{\gamma_{1}}\right| \geqslant e^{-2 \hat{H}}
$$

since $\left|\gamma_{1}\right| \leqslant 2 \hat{H}$. Therefore, we have

$$
1 \leqslant|L|(r !)^{s} \hat{H}^{8 s^{2}(s-1) \hat{D}^{2} N} e^{2 \hat{H}}+\frac{1}{2} .
$$

From $(34)$ and $N \leqslant(s+1) r$, we obtain

$$
|L| \geqslant \frac{1}{2}(r !)^{-s} \hat{H}^{-8 s^{2}(s-1) \hat{D}^{2} N} e^{-2 \hat{H}} \geqslant \exp \left(-\hat{H}^{10 s^{4} \hat{D^{2}}}\right) .
$$

Hence it follows immediately by (17) and (20) that

$$
|L| \geqslant \exp \left(-\frac{1}{3} c_{1} H^{c_{2}}\right),
$$

where $c_{1}=\exp \exp \left(\tau k^{2} d^{k+1} A\right)$ and $c_{2}=\exp \left(\tau k^{2} d^{k+1} A\right)$. Here, recalling the equality (15) and using the estimate (16), we have

$$
\left|\sum_{j=1}^{m} B_{j} e^{\beta_{j}}\right|>\exp \left(-\frac{1}{2} c_{1} H^{c_{2}}\right) \text {. }
$$

Finally, recalling the equality (7) and using the estimate (8), we obtain

$$
\left|\sum_{i=1}^{k} A_{i} e^{a_{i}}\right|>\exp \left(-c_{1} H^{c_{2}}\right)
$$

This concludes the proof of the theorem.

\section{References}

[1] A. O. Gelfond, Transcendental and algebraic numbers, Dover, New York 1960.

[2] S. Lang, A transcendence measure for E-functions, Mathematika 9 (1962), pp. $157-161$. 
[3] K. Mahler, Zur Approximation der Exponentialfundition und des Logarithmus $I$ J. Reine Angew. Math. 166 (1932), pp. 118-136.

[4] - On a paper by A. Batier on the approximation of rational powers of e, Aota Arith. 27 (1975), pp. 61-87.

[5] C. L. Siegel, Transcendental numbers, Annals of Math. Studies 16, Princeton 1949.

DEPARTMANT OF MLATHEMATICS, FACUTLTY OF SCTENOH IBARAKT UNIVERSITY

Anwendung einer Summationsformel auf Dirichletsche Reihen und verallgemeinerte Dedekindsche Summen

von

1. Einleitung. Bereits Dedekind [2] bewies für die klassischen Dedekindschen Summen

$$
s(h, k)=\sum_{\mu(\bmod k)}\left(\left(\frac{\mu}{k}\right)\right)\left(\left(\frac{h \mu}{k}\right)\right)
$$

mit $h, k \in \mathbf{N}$

$$
((x))= \begin{cases}x-[x]-\frac{1}{2}, & x \notin Z \\ 0, & x \in Z\end{cases}
$$

die Gleichung

(1) $s(p h, k)+\sum_{m=0}^{p-1} s(h+m k, p h)=(p+1) s(h, k)$,

wobei $p$ eine Primzahl ist. Diese Identität ist ein Spezialfall des PeterssonKnopp Theorems $\left(^{1}\right)$ (Knopp [4]):

Frür $h, k, n \in Z, k>0, n>0,(h, k)=1$ ist

$$
\sum_{\substack{(a, d) \in N^{2} \\ a \cdot d=n}} \sum_{b(\bmod d)} s(a h+b l, d k)=\left(\sum_{\substack{m \in N \\ m[n}} m\right) s(h, k)
$$

Parson und Rosen [5] bewiesen ein analoges Resultat für verallgemeinerte Dedekindsche Summen:

Setzt man $\bar{B}_{r}(x)=B_{r}(x-[x]), r \in N \cup\{0\}$, wobei $B_{r}(y)$ die durch

$$
\frac{\approx e^{y z}}{e^{z}-1}=\sum_{r=0}^{\infty} B_{r}(y) \frac{z^{r}}{r !}
$$

( $\left.{ }^{1}\right)$ Diese Bezeichnung stammt ron Goldberg [3]. 